\title{
Small strange stars and marginally stable orbit in Newtonian theory
}

\author{
Julian Leszek Zdunik \\ N. Copernicus Astronomical Center, Polish Academy of Sciences, Bartycka 18, PL-00-716 Warszawa, Poland, \\ e-mail: jlz@camk.edu.pl \\ Eric Gourgoulhon \\ Département d'Astrophysique Relativiste et de Cosmologie - UMR 8629 du CNRS, Observatoire de Paris, \\ F-92195 Meudon Cedex, France, e-mail: Eric.Gourgoulhon@obspm.fr
}

(15 December 2000)

\begin{abstract}
It is shown that for very rapidly rotating low mass strange stars the marginally stable orbit is located above the stellar surface. This effect is explained by the very important role of the oblateness of the rotating strange star. The comparison with some "academic" examples is presented. This feature is purely Newtonian in its nature and has nothing to do with relativistic marginally stable orbit. The effect is very large and cannot be treated in a perturbative way. It seems that strange stars as a very dense self-bound objects are the only possibility in Nature to represent these toy models.
\end{abstract}

04.40.Dg, 95.30.Sf

Recently marginally stable orbits have been extensively studied as a crucial input of the most popular model explaining kilohertz quasi-periodic oscillations (QPOs) in X-ray binaries (see [1] for a review). Besides neutron stars as a possible source of these QPOs hypothetical strange stars have been considered. Strange stars, objects built of the mixture of $\mathrm{u}, \mathrm{d}, \mathrm{s}$ quarks, in contrast to neutron stars have no minimum mass; their size extends continuously from that of large nuclei (strangelets) up to the maximum mass configurations of about 2 solar masses (for a recent review, see [2]). It has been showed [3, 4h that the marginally stable orbit is located above the surface of the strange star for relatively high mass even at very high rotation rates, which is the main difference with respect to neutron stars [5]. It was suggested [4] that, for configurations close to the Keplerian one, defined as a mass-shedding limit at the equator, the very large deformation of the strange star leads to the significant increase of the radius of the marginally stable orbit, resulting in a gap between this orbit and the stellar surface. Of course in these considerations, performed for relatively massive $\left(M>\mathrm{M}_{\odot}\right)$ stars, the GR effects seem to play a dominant role. General relativity predicts that the radius of the marginally stable orbit for nonrotating star of mass $M$ is equal to $\approx 8.8 M / \mathrm{M}_{\odot} \mathrm{km}$, which is comparable to the radius of a strange star. It is well known that quadrupole moment of the star could also destabilize the orbital motion [6], but it seemed that this effect is not very large in real physical situations, at least as far as neutron stars are concerned. We show that for objects such as self-bound strange stars the deformations due to the rotation can be so large that the change in Newto- nian gravitational potential could result in the existence of a marginally stable orbit, even when GR corrections are completely negligible.

The existence of a marginally stable orbit corresponds to the conditions $\mathrm{d} V / \mathrm{d} r=0$ and $\mathrm{d}^{2} V / \mathrm{d} r^{2}=0$ where $V(r)$ is the effective potential (for the radial part of motion) for a particle orbiting the central body at a fixed angular momentum per unit mass $l$. The resulting equations are discussed in standard textbooks in the case of the Schwarzschild geometry (see e.g. [7]). In first approximation, the source of the marginally stable orbit is the term in $V$ proportional to $1 / r^{3}$ with the same sign as the gravitational potential (i.e. opposite sign to centrifugal force). In general relativity it is a natural result of constants of geodesic motion on circular orbits.

In Newtonian theory this term is generated by the quadrupole moment of the star $Q$; in the equatorial plane the gravitational force per unit mass of the orbiting particle is

$$
f_{g}=-\frac{G M}{r^{2}}-\frac{3 Q}{2 r^{4}}
$$

With the above convention $Q>0$ for an oblate star and $Q<0$ for a prolate one. The relativistic definition of $Q$ can be found in Ref. [8].

One can take into account the effect of the oblateness and of the angular momentum of the star using standard methods, i.e. via expansions of the metric functions in powers of $1 / r^{2}$. In the framework of GR this leads to the following formula for marginally stable orbit:

$$
\begin{aligned}
\frac{R_{\mathrm{ms}}}{3 R_{\mathrm{g}}}= & \frac{1}{2}\left(1+\sqrt{1+q_{\mathrm{GR}} / 6}\right)- \\
& \left(\frac{2}{3}\right)^{3 / 2} j \sqrt{1+\frac{9}{16}\left(\sqrt{1+q_{\mathrm{GR}} / 6}-1\right)},
\end{aligned}
$$

where $q_{\mathrm{GR}}=Q c^{4} / G^{2} M^{3}, j=J c / G M^{2}, R_{\mathrm{g}}=2 G M / c^{2}$, $J$ being the total angular momentum and we linearized the dependence in $j$. In the case of $Q=0 \mathrm{Eq}$. (2) reduces to the formula derived by Kluzniak and Wagoner [9].

In nonrelativistic limit we have

$$
R_{\mathrm{ms}} \rightarrow \frac{3}{2}\left(R_{\mathrm{g}}+\sqrt{R_{\mathrm{g}}^{2}+\frac{2 Q}{3 M}}\right) \rightarrow \sqrt{\frac{3 Q}{2 M}} .
$$

In Newtonian theory the marginally stable orbit can be determined exactly for some toy models. We will present here results for one- and two-dimensional cases. 
In one dimension we consider a bar with given constant linear density $\rho_{l}$, i.e. mass of the bar is $M=2 \rho_{l} R$, where $R$ is the "radius" of the bar equal to the half of its length. The gravitational attraction on the line which coincides with the bar at the distance $r$ from the bar center is equal to $f_{g}=-G M /\left(r^{2}-R^{2}\right)$. Of course we have to assume that this field does not change during orbital motion of the particle, i.e. the bar rotates with the same angular velocity as the orbiting particle. The radius of the marginally stable orbit is then

$$
R_{\mathrm{ms}}^{\mathrm{bar}}=\sqrt{3} R \simeq 1.73 R .
$$

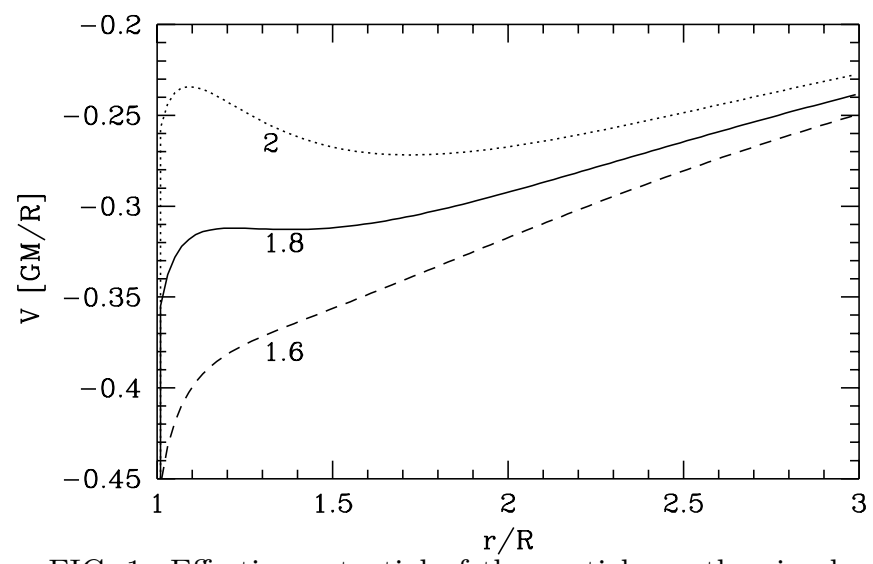

FIG. 1. Effective potential of the particle on the circular orbit around the disk when the square of angular momentum $l^{2}$ is equal to $1.6,1.8$ and $2 G M R$. The solid line corresponds to the case of marginally stable orbit (the exact value is $l_{\mathrm{ms}}^{2}=1.784 G M R$ ).

In two dimensions the corresponding body is an infinitesimally thin disk of constant surface density $\rho_{s}$ with mass $M=\pi \rho_{s} R^{2}$. The gravitational force in the plane of the disk at the distance $r$ is then given by the hypergeometric function ${ }^{2} F_{1}$

$$
f_{g}=-\frac{G M}{r^{2}} \frac{1}{(1+R / r)^{3 / 2}}{ }^{2} F_{1}\left(\frac{3}{4}, \frac{5}{4}, 2 ; q^{2}\right),
$$

where $q=2 r R /\left(r^{2}+R^{2}\right)$. The form of the effective potential [the integral of Eq. (5) plus a centrifugal term] results in the marginally stable orbit located at the radius

$$
R_{\mathrm{ms}}^{\text {disk }} \simeq 1.2885 R
$$

The angular momentum of the particle at this orbit is then equal to $l \simeq \sqrt{1.784 G M R}$ (per particle mass).

It should be mentioned that in these examples expansions of the gravitational potential in powers of $(R / r)^{2}$ would be completely unjustified. For example in the case of a disk the expansion of Eq. (5) leads to the formula:

$$
f_{g}=-\frac{G M}{r^{2}}\left(1+\frac{3}{8}(R / r)^{2}+\frac{15}{64}(R / r)^{4}+\ldots\right) .
$$

Using only the second term (quadrupole order) we obtain the radius of marginally stable orbit

$$
R_{\mathrm{ms}}^{\mathrm{disk}}[Q]=\sqrt{0.375} R \simeq 0.61 R
$$

i.e more than twice smaller than the exact value.

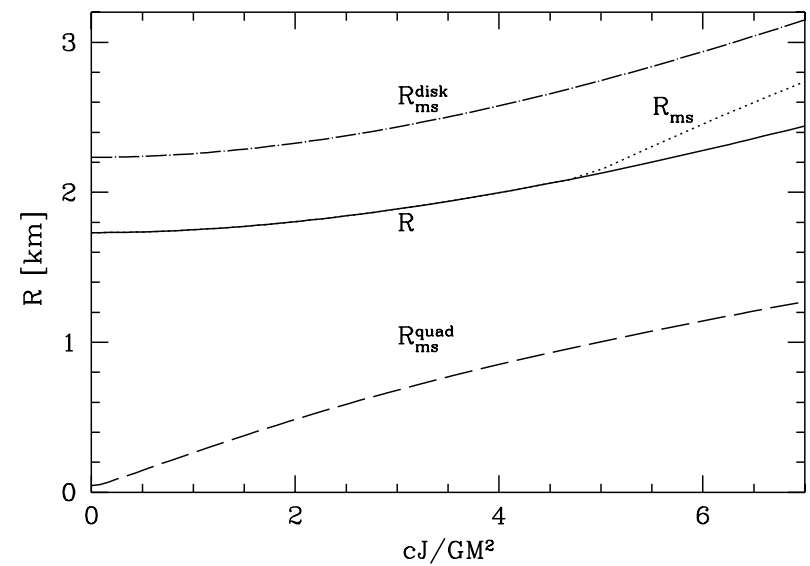

FIG. 2. Equatorial radius of the strange star $R$ (solid), radius of the marginally stable orbit $R_{\mathrm{ms}}$ (dotted), quadrupole approximation of $R_{\mathrm{ms}}-R_{\mathrm{ms}}^{\text {quad }}$ (dashed), and radius of the marginally stable orbit if the rotating object would be a disk of the same radius $R_{\mathrm{ms}}^{\text {disk }}$. The star has a mass $0.005 \mathrm{M}_{\odot}$. The value of $R_{\mathrm{ms}}^{\text {quad }}$ at $J=0$ corresponds to the relativistic radius of the marginally stable orbit for nonrotating star which in this case is equal to $0.044 \mathrm{~km}$. The relativistic term due to the dependence on $j$ (Eq. Q) is negligible.

In previous papers [3, 4 the marginally stable orbit for rotating strange stars have been determined in the framework of General Relativity and the authors concluded that the main difference with respect to neutron stars is the gap between this orbit and the stellar surface for high rotation rates.

Rotating strange stars are very oblate for large rotation rates and the Keplerian configurations for a sequence of stars with given baryon number is achieved not due to the increase of the rotation frequency $\nu_{\text {rot}}$, but due to the deformation of the star [4. It was suggested that this large oblateness is the true reason which cause the radius of the marginally stable orbit to be above the stellar surface.

In this paper we try to prove this statement by considering strange stars for which relativistic effects are very small. Thus we will focus on the results for small strange stars where the oblateness is crucial for the properties of these objects and in particular, is the source of the marginally stable orbit. The effects of General Relativity, which are very important in massive dense stars, are here negligible. However presented results has been obtained in the framework of GR using the numerical code developed by Gourgoulhon et al. 10].

We calculate rotating bare strange stars (without crust) using the strange matter model considered as a basic one in previous papers (and called SQM1 in [4]).

In Fig. 22 we present the equatorial radius of the star $R_{\text {eq }}$ with fixed baryon mass $M=0.005 \mathrm{M}_{\odot}$ and the radius of marginally stable orbit. We choose such a small 
object in order to extract Newtonian effects - which for massive stars are contaminated (and for slowly rotating stars dominated) by relativistic ones. We see that for high rotation rates there exist a gap between stellar surface and the marginally stable orbit. The curve $R_{\mathrm{ms}}^{\text {disk }}$ is defined by Eq. (6) with $R=R_{\text {eq }}$. The curve $R_{\mathrm{ms}}^{\text {quad }}$ corresponds to the quadrupole approximation of $R_{\mathrm{ms}}$ given by Eq. (2) with $Q$ calculated in the framework of GR [Eq. (7) of [8]. We see that this quadrupole approximation is wrong and as in the case of disk the resulting radius is about twice smaller than the true value. This conclusion confirms the results of Shibata and Sasaki [6], who found necessary to include higher multipole moments in the marginally stable orbit determination in the case of polytropic EOS. They concluded that the quadrupole moment is as important as the spin. In our model the effect of the rotational deformation of the star is larger because the matter is self-bound at very high density and the stars are more oblate. Due to these two reasons for fast rotating strange stars we are "closer" to the disk approximation than to the expansion up to the quadrupole term (see Fig. 2). The value of the quadrupole moment for small strange stars close to the Keplerian configuration is $Q_{\max }=0.17 M R^{2}$ which is quite close to the "disk" value $-0.25 M R^{2}$.
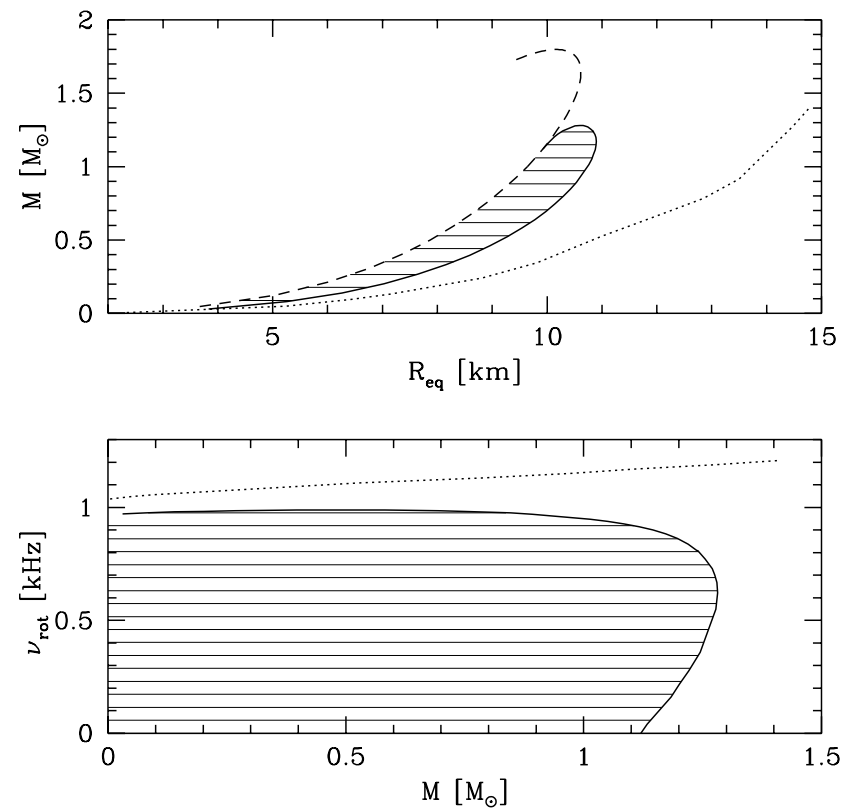

FIG. 3. The regions where equatorial radius of the marginally stable orbit is smaller than stellar radius (hatched). Solid curve is defined by the equality $R_{\mathrm{eq}}=R_{\mathrm{ms}}$. The condition $R_{\mathrm{eq}}<R_{\mathrm{ms}}$ is fulfilled between solid and dotted curve (corresponding to the Keplerian frequency). The dashed curve represents nonrotating configurations (top panel). In hatched area the ISCO is defined by the stellar surface. The region for $1.12<M / \mathrm{M}_{\odot}<1.28$ corresponds to the configurations for which for given mass $R_{\mathrm{ms}}$ decreases with $\nu_{\text {rot }}$ due to dependence on $j$, then crosses the radius and for higher $\nu_{\text {rot }}$ reappears.
The behavior of $R_{\mathrm{eq}}$ and $R_{\mathrm{ms}}$ for a star rotating faster and faster, presented in Fig. 2, is typical for small mass stars self-bound at high density. For higher masses (larger than $1.12 \mathrm{M}_{\odot}$ in this model) the radius of marginally stable orbit is larger than $R$ at $\nu_{\text {rot }}=0$ and for low rotation rates decreases due to the relativistic effects and then increases due to the oblateness (see |14 for figures and discussion). Of course the point at which $R_{\mathrm{ms}}\left(\nu_{\mathrm{rot}}\right)$ gets larger than the equatorial radius $R_{\text {eq }}$ depend on the mass of the star under consideration.

In the next figures we present the regions of stellar parameters and rotation rates for which marginally stable orbit is located above the stellar surface.

In Fig. 3 we show the boundaries resulting from the Keplerian frequency and from the condition $R_{\mathrm{eq}}=R_{\mathrm{ms}}$. We see that in $M-R$ plane the region with $R_{\mathrm{ms}}<R_{\mathrm{eq}}$ looks rather small and even for low-mass stars there exist relatively large area in which $R_{\mathrm{eq}}<R_{\mathrm{ms}}$. It is also clearly visible that the stars with masses between 1.12 and $1.28 \mathrm{M}_{\odot}$ enter the region with $R_{\mathrm{ms}}<R_{\mathrm{eq}}$ during slowing down or acceleration although for high and low rotation rates $R_{\mathrm{ms}}>R_{\mathrm{eq}}$. These stars for fixed baryon mass are represented by nearly horizontal (vertical) lines in top (bottom) panel of Fig. 3 ("nearly" because gravitational mass increases a little due to the rotation).

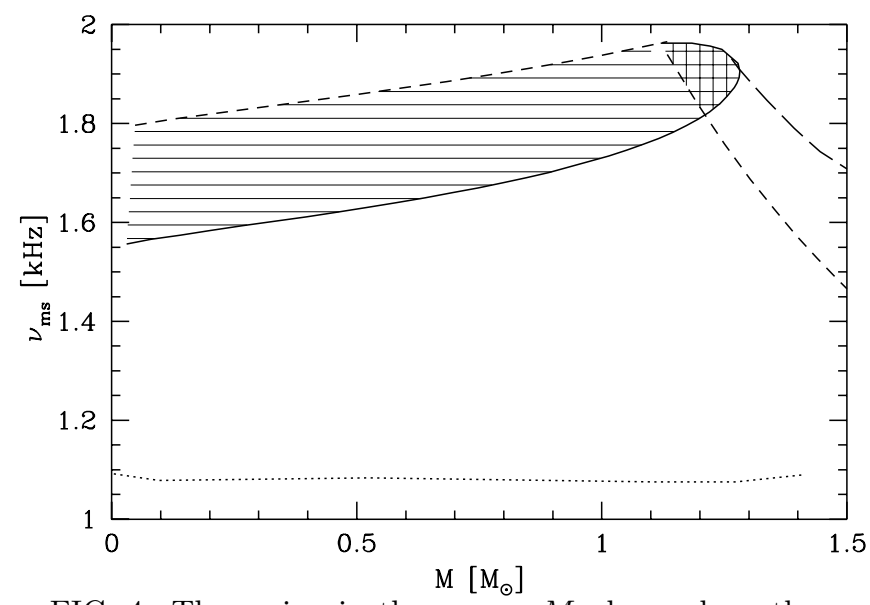

FIG. 4. The region in the $\nu_{\mathrm{ms}}$ vs $M$ plane where the radius of the marginally stable orbit is smaller than the stellar radius (hatched area). In the double hatched region there exist configurations with $R_{\text {eq }}<R_{\mathrm{ms}}$ (slowly rotating) as well as $R_{\text {eq }}<R_{\text {ms }}$ (see text). The solid curve is defined by the condition $R_{\mathrm{eq}}=R_{\mathrm{ms}}$, the dashed line is the frequency at the ISCO for nonrotating star, which for $M<1.12 \mathrm{M}_{\odot}$ coincides with stellar radius and for higher masses is proportional to $1 / M$. The long dashed curve is the maximum $\nu_{\mathrm{ms}}$ for higher mass strange stars when $R_{\text {eq }}<R_{\mathrm{ms}}$ everywhere. The dotted curve is the minimum value of $\nu_{\mathrm{ms}}$ achieved for Keplerian configurations.

In Fig 1 we present limits on the frequency of marginally stable orbit taking into account the conditions $R_{\text {eq }}<R_{\mathrm{ms}}$ which in principle can be supported by the observations of QPOs [11]. 
There exists a quite large region in the $\nu_{\mathrm{ms}^{-}} M$ plane, where this condition is fulfilled. It should be mentioned that because $\nu_{\mathrm{ms}}$ in non-monotonic function of $J$ for a star with given baryon mass, one region in $\nu_{\mathrm{ms}}-M$ plane is not uniquely defined. Namely in double hatched area there exist stellar configurations with $R_{\text {eq }}<R_{\mathrm{ms}}$ (slowly rotating) as well as stars for which $R_{\mathrm{eq}}>R_{\mathrm{ms}}$. For nonrotating stars we have here $R_{\mathrm{eq}}<R_{\mathrm{ms}}$ but if such a star rotates faster and faster it moves upwards, turns back at the solid line and moves downwards having $R_{\mathrm{eq}}>R_{\mathrm{ms}}$ as long as it do not leave the region between solid lines. One has similar situation (non-uniqueness) in the case of high mass stars $\left(M>1.28 \mathrm{M}_{\odot}\right)$, which turn back at longdashed line but the condition $R_{\mathrm{eq}}<R_{\mathrm{ms}}$ is fulfilled all the time. In non hatched region all stars have $R_{\mathrm{eq}}<R_{\mathrm{ms}}$.

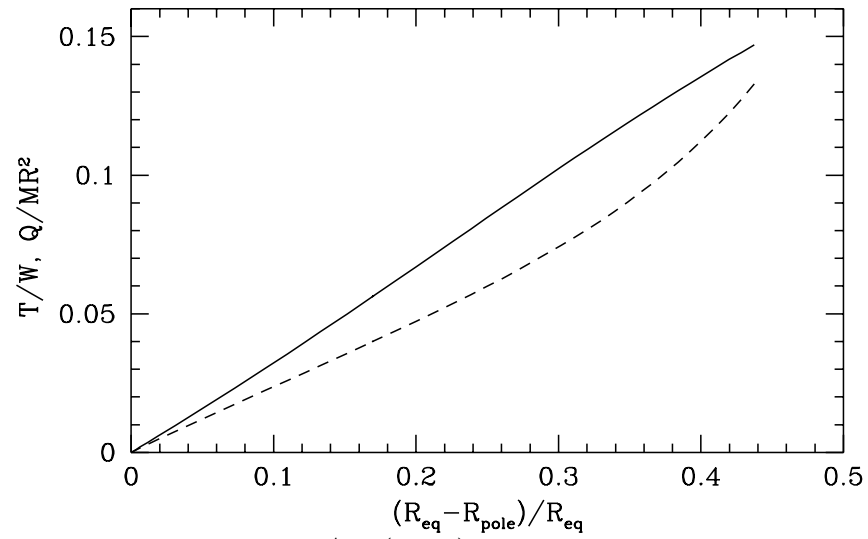

FIG. 5. The ratio $T / W$ (solid) and the quadrupole moment $Q$ (dashed) as a function of the oblateness of the star along the sequence of rotating strange stars for which $R_{\mathrm{eq}}=R_{\mathrm{ms}}$ (solid curves in Figs 3). Point $(0,0)$ describes nonrotating configuration. The last points (maxima) correspond to the smallest stars. The Keplerian limits are located far above and to the right of this graph $(T / W \sim 0.25$, oblateness $\sim 0.63)$

From Fig. 5 we see that the oblateness of the star, the quadrupole moment $Q$ and the ratio of the kinetic to the potential energy $T / W$ for the rotating stellar configurations defined by the equality $R_{\mathrm{eq}}=R_{\mathrm{ms}}$ are highly correlated and almost linearly dependent. Although the frequency at which this condition is fulfilled is approximately constant for $M<1 \mathrm{M}_{\odot}(0.9-1 \mathrm{kHz}$, Fig 3) the stars with lower mass are much more oblate at this rotation rate, having proportionally larger $T / W$ and $Q$. For these configurations large value of $R_{\mathrm{ms}}$ results entirely from oblateness of the star. Albeit the role of the oblateness for the location of the marginally stable orbit is most pronouncing in the case of small strange stars close to the Keplerian velocity, also for massive stars rotating with relatively high frequency the deformation of the star is crucial for the existence of the marginally stable orbit.

In this paper we showed that the gap between innermost stable orbit and the surface of the star can be produced by the oblateness of the star in the framework of Newtonian theory. Due to the compactness and very high surface density strange stars are natural candidates for this effect to be a real possibility in Nature. This is due to two reasons. First: the stars are very oblate, and the gravitational force is far from the attraction by a point mass. Second: the stars are self-bound at very high density and in practice an oblate small strange star is quite close to a constant density disk. In contrast to bare strange stars, for oblate neutron stars (and also for strange stars with crust) the low-density region is relatively large making the radius of the star larger than $R_{\mathrm{ms}}$ (or in other words the radius of corresponding "disk" is much smaller than the stellar radius).

\section{ACKNOWLEDGMENTS}

We are very grateful to P. Haensel for careful reading of manuscript and helpful comments and suggestions. This research was partially supported by the KBN grants No. 2P03D.014.13. The numerical calculations have been performed on computers purchased thanks to a special grant from the SPM and SDU departments of CNRS. Main results reported in the present note were obtained during visit of one of the authors (J.L.Z) at DARC, Observatoire de Paris- Meudon, in July 2000, within the framework of the CNRS/PAN program Jumelage Astrophysique.

[1] M. van der Klis, Annu. Rev. Astron. Astrophys. 38, 717 (2000).

[2] J. Madsen, in Hadrons in Dense Matter and Hadrosynthesis Lecture Notes in Physics, edited by J. Cleymans (Springer-Verlag, 1999), p. 162; N.K. Glendenning, Compact stars (Springer, New York, 1997)

[3] N. Stergioulas, W. Kluźniak, and T. Bulik, Astron. Astrophys. 352, L116 (1999).

[4] J. L. Zdunik, P. Haensel, D. Gondek-Rosińska, and E. Gourgoulhon, Astron. Astrophys. 356, 612 (2000).

[5] M. C. Miller, F. K. Lamb, and G. B. Cook, Astrophys. J. 509, 793 (1998).

[6] M. Shibata and M. Sasaki, Phys. Rev. D 58, 104011 (1998).

[7] C. W. Misner, K. S. Thorne, and J. A. Wheeler, "Gravitation" (W.H. Freeman and Co., San Francisco, 1973), p. 660 .

[8] M. Salgado, S. Bonazzola, E. Gourgoulhon, and P. Haensel, Astron. Astrophys. 291, 155 (1994).

[9] W. Kluzniak and R. V. Wagoner, Astrophys. J. 297, 548 (1985).

[10] E. Gourgoulhon et al., Astron. Astrophys. 349, 851 (1999).

[11] P. Kaaret et al., Astrophys. J. Lett. 520, L37 (1999). 